\title{
Model of smoothing roughness on GaAs wafer surface by using nonabrasive chemical-and-mechanical polishing
}

\author{
A.V. Fomin, G.A. Pashchenko, M.Yu. Kravetskyi, I.G. Lutsyshyn \\ V. Lashkaryov Institute of Semiconductor Physics, NAS of Ukraine, \\ 45, prospect Nauky, 03028 Kyiv, Ukraine \\ E-mail:ipsfomin@ukr.net
}

\begin{abstract}
Studied experimentally in this work has been the process of smoothing relief roughness on the GaAs wafer surface by using the method of contactless nonabrasive chemical-and-mechanical polishing under conditions of its rotational movement relatively to the polishing plate. The model of this process has been developed under assumption that the pure diffusion smoothing mechanism takes place there. It has been shown that satisfactory correspondence between respective calculated dependences and experimental results can be reached by introducing the "effective" diffusion coefficient providing account of etchant convection.
\end{abstract}

Keywords: contactless chemical-and-mechanical polishing, gallium arsenide, diffusion layer, convection.

Manuscript received 22.11.16; revised version received 01.02.17; accepted for publication 01.03.17; published online 05.04.17.

\section{Introduction}

The essence of method for contactless chemical-andmechanical polishing (CCMP) consists in using the effect of smoothing roughness due to a small thickness of the etching liquid layer between surface of the polished wafer and moving polishing plate. The thickness of this layer is comparable with the depth of smoothed roughness, therefore, the density of etchant diffusion flow to asperities is higher, and, consequently, the speed of etching them is much higher than the speed of etching the surface valleys.

Earlier in [1], the authors studied the process of treating semiconductor wafers by using the CCMP method in the regime of a fixed stationary wafer, the surface of which was characterized by relief two-dimensional extended defects (scratches). It was ascertained that, for morphological defects oriented in longitudinal and transversal directions relatively to incoming flow, the speeds of smoothing are different, which is caused by differences in local convection conditions.

In a real CCMP process, the treated wafer is in the rotational movement relatively to the polishing plate. In relation with it, orientation of linear irregularities relatively to incoming etchant flow is permanently changed, and etchant convection has more complex character. One can assume that the latter resembles chaotic diffusion transfer. It would allow describing the process that provides smoothing these irregularities on the wafer surface by using the model for pure diffusion smoothing mechanism but introducing the "effective" diffusion coefficient providing account of convection. So, it is interesting to compare experimental and calculated relations inherent to smoothing the linear irregularities by using the CCMP method, which will enable to ascertain validity of the offered way to account for contribution of a convective component to the real shape-changing process. 
Therefore, it is of interest to ascertain appropriateness of this approach to account for contribution of the convective component to the real process of shape changing when applying the CCMP method by comparing the respective calculated dependences with the results of experimental investigations.

\section{Experimental}

Experimental studying the CCMP process was carried out using the wafers of monocrystalline GaAs (100). As an etchant, we used the solution of bromine in hydrobromic acid with the concentration $2 \%$ $\left(0.06 \mathrm{~g} / \mathrm{cm}^{3}\right)$, the linear velocity of the polishing plate in the field of treated wafer was $7 \mathrm{~cm} / \mathrm{s}$. At the beginning of experiments, the initial wafer surface was polished for the period necessary to form its stationary shape. To be convinced that the wafer surface shape is stabilized, we used the profilograph-profilometer (ЛОМО, model 252) every $5 \mathrm{~min}$ of polishing to take profilograms for comparison with the previous ones.

After reaching the stationary shape of surface, using the wire saw it was made a kerf imitating the linear defect of relief. Then polishing was continued. After each 5-min cycle of smoothing, we used the measuring head МИГ-1 to determine the mean thickness of the layer material removed from the wafer surface and, using the micro-interferometer МИИ-4, the depth of kerf in several fixed points.

\section{Results and discussion}

It can be shown that the one-dimensional model of pure diffusion smoothing implies the following formula for the dependence of valley depth on the polished surface on duration of the CCMP process [2]:

$t=\frac{\Delta^{2} \rho}{\beta D c_{0}} \ln \frac{h_{0}}{h}+\frac{\Delta \rho}{\beta D c_{0}}\left(h_{0}-h\right)$,

where $\Delta$ is the thickness of the diffusion layer, inside which the concentration changes from $c_{0}$ at its boundary to zero at the wafer surface (Fig. 1), $h_{0}$ and $h$ are initial and current (i.e., to the time $t$ ) valley depths, respectively, $D$ is the diffusion coefficient, $\beta$-maximum mass of dissolved material that can be absorbed by the unit mass of this etchant, $\rho$ - density of dissolved material.

In the formula (1), to take into account the influence of convection by introducing the "effective" diffusion coefficient, one should appreciate that convection contribution to the transfer process should monotonically tend to zero when coming nearer to the reaction surface [2]. The simplest formula for such an "effective" diffusion coefficient suitable for calculations of the etching rate for surface asperities and valleys has the following look:

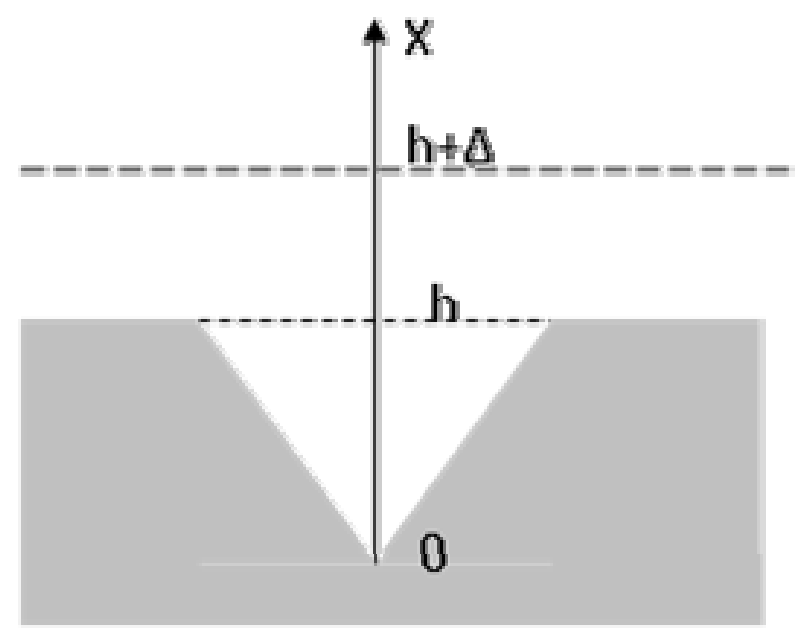

Fig. 1. Model for smoothing the valley on the crystal surface in the CCMP process.

$$
\begin{aligned}
& D_{e f f}=D+K\left(1-\frac{x}{\Delta}\right), \\
& D_{e f f}=D+K\left(1-\frac{x}{\Delta+h}\right),
\end{aligned}
$$

where $K$ is the "convection coefficient" constant for the set experimental conditions, $x$ - distance from the given point to the diffusion layer boundary (Fig. 1). As a consequence, the density of stationary convectivediffusion flow of the etchant to surface asperity is equal:

$$
j_{1}=-D_{e f f} \frac{d c}{d x}=-\left[D+K\left(1-\frac{x}{\Delta}\right)\right] \frac{d c}{d x}
$$

where $c$ is the concentration of reacting particles, for which the following formula is valid:

$$
c=-j_{1} \int \frac{d x}{D+K\left(1-\frac{x}{\Delta}\right)}+\text { const }
$$

or

$$
c(x)=j_{1}\left[\frac{\Delta}{K} \ln \left(D+K-\frac{K}{\Delta} x\right)+\text { const }\right] .
$$

Let us assume that the etchant concentration $c=0$ at the reaction surface when $x=\Delta$. Then, as it follows from the formula (4)

const $=-\frac{\Delta}{K} \ln D$ 
It follows thereof and from the formula (4) that at the diffusion layer boundary for $x=0$ and $c=c_{0}$

$c_{0}=j_{1} \frac{\Delta}{K} \ln \left(\frac{D+K}{D}\right)$.

Thus, for the density of the etchant stationary convective-diffusion flow $j_{1}$ to a surface asperity, the following expression is valid:

$j_{1}=\frac{K c_{0}}{\Delta \ln \frac{D+K}{D}}$.

By analogy, one can show that the density of the etchant stationary convective-diffusion flow $j_{2}$ to a surface valley is determined by the formula

$$
j_{2}=\frac{K c_{0}}{(\Delta+h) \ln \frac{D+K}{D}} .
$$

Therefore, the speed of smoothing is as follows:

$$
\frac{d h}{d t}=\frac{\beta}{\rho}\left(j_{2}-j_{1}\right)=-\frac{\beta K c_{0}}{\rho \ln \frac{D+K}{D}}\left(\frac{1}{\Delta}-\frac{1}{\Delta+h}\right) .
$$

As a result of integrating the formula (6), one can obtain the expression $t=\frac{\rho \Delta^{2}}{\beta K c_{0}} \ln \frac{D+K}{D} \ln \frac{h_{0}}{h}+\frac{\rho \Delta}{\beta K c_{0}} \ln \frac{D+K}{D}\left(h_{0}-h\right)$,

which can be also obtained directly from the formula (1) by changing the diffusion coefficient $D$ in it with the expression for $D^{*}$ :

$$
D^{*}=\frac{K}{\ln \frac{D+K}{D}}
$$

It is of practical interest how the depth $h$ of smoothed irregularity depends on the thickness $H$ of material etched from surface. The dependence of $H$ on the etching time $t$ obtained from the formula (5) looks like that:

$$
H=\frac{\beta}{\rho} \frac{K c_{0}}{\Delta \ln \frac{D+K}{D}} t
$$

Thereof and from the formula (7), one can obtain the following expression:

$H=\Delta \ln \frac{h_{0}}{h}+\left(h_{0}-h\right)$

The obtained dependence is shown in Fig. 2 as a function $h(H)$ for some set value of diffusion layer $\Delta=45 \mu \mathrm{m}$ that provides the best correspondence with the experimental data. It is noteworthy that the formula

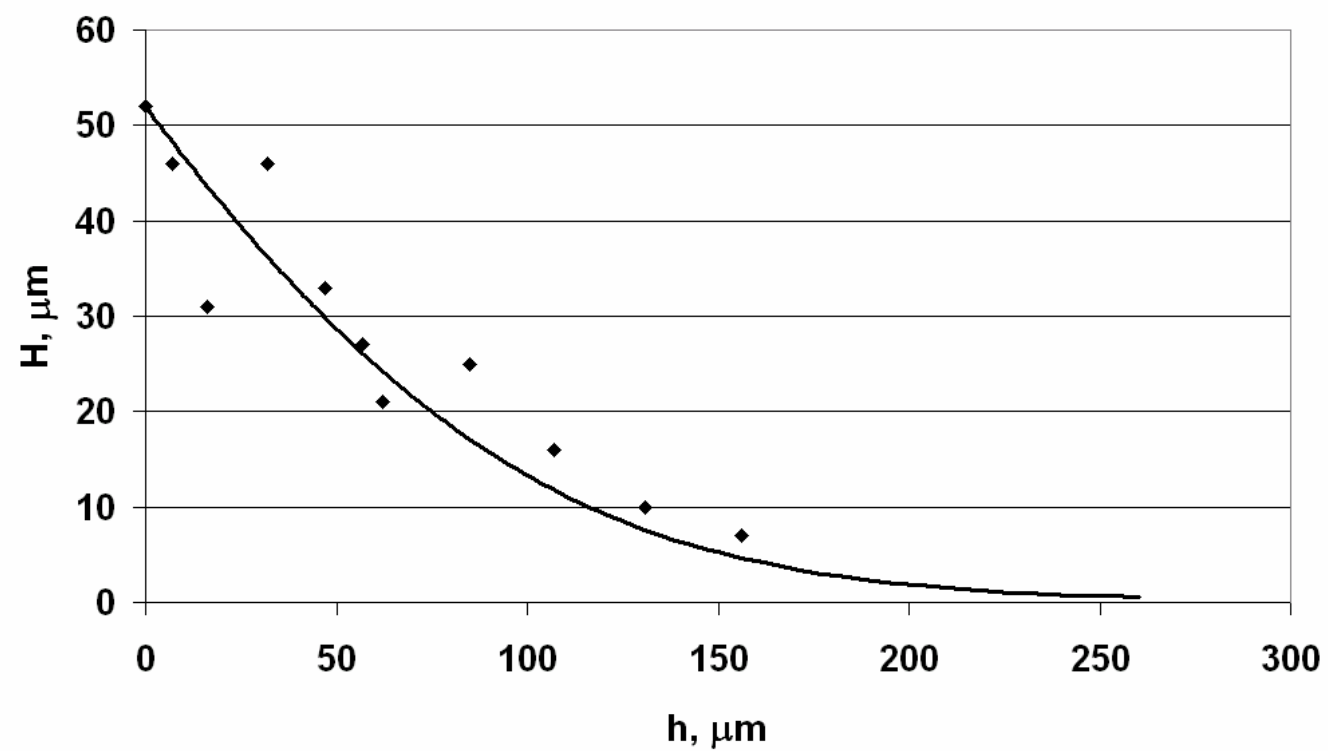

Fig. 2. Dependence of the smoothed irregularity depth on the thickness of material etched off the surface. 
(9) is valid independently of taking convection into account. As seen from Fig. 2, for a given $\Delta$ value, to provide total smoothing the irregularity of the depth approximately $50 \mu \mathrm{m}$, it is necessary to etch off about $250 \mu \mathrm{m}$. The formula (9) shows that, when smoothing the surface, material losses are decreased with the $\Delta$ value.

The expected order of the irregularity smoothing rate can be estimated before experiments by using the formulae (1) and (7), since the values of available there parameters (or, at least, their orders) are either known or can be determined experimentally for the used conditions.

For instance, the $\beta$ parameter value was determined in the following way. Into a container filled with a known amount of etchant, we placed the preliminary weighed sample of the dissolved material possessing the mass $m_{0}$. Then, the container was sealed, and the sample in it was dissolved for several hours. After that, the sample was extracted from the container, dried and weighed again. Then, it was placed into the same vessel with the same etchant, and the procedure was repeated up to the moment when the result of another weighing $m_{n}$ begins to repeat the previous one, which was indicative of stopping the dissolution process. After that, the $\beta$ parameter value was determined using the following formula:

$$
\beta=\frac{m_{0}-m_{n}}{m^{\text {etch }}}
$$

where $m^{\text {etch }}$ is the initial mass of the etchant active component. The $\beta$ value determined using the above technique is close to 0.05 .
In accord with calculations based on the theory of convection- diffusion transfer [2], when the space between the polished surface and polishing plate is lower than $50 \mu \mathrm{m}$, the etchant concentration above the larger part of the sample surface is, at least, $30 \%$ lower than the initial value $c_{0}$ even near the polishing plate surface. It means that the diffusion layer comprises the whole thickness of this space.

Since the etchant is quickly exhausted, its concentration above the kerf region is essentially lower than the initial one. Therefore, to calculate using the formulae (1) and (7), the $c_{0}$ value was taken as $0.03 \mathrm{~g} / \mathrm{cm}^{3}$. The diffusion coefficient was equal to $10^{-5} \mathrm{~cm}^{2} / \mathrm{s}$. At last, the thickness of the convectivediffusion layer $\Delta$, as it was mentioned above, can be determined using the formula (9), being based on the experimental data presented in Fig. 2.

Shown in Fig. 3 are the experimental and calculated dependences $h(t)$ without account of convection (curve 1) and with it (curve 2).

When $\Delta=45 \mu \mathrm{m}$, the initial speed of smoothing the irregularity in accord with the formula (1), without account of convection, is approximately $0.2 \mu \mathrm{m} / \mathrm{min}$, while the speed of material removal is close to $0.5 \mu \mathrm{m} / \mathrm{min}$, which is 4 to 5 times lower than the experimental value.

Thus, to coordinate the results of calculations with the experimental data is possible only when using the additional parameter $K$ defining the convection intensity. $K$ value providing maximum matching with experimental data is equal to $1.4 \cdot 10^{-4} \mathrm{~cm}^{2} / \mathrm{s}$ (Fig. 3, curve 2$)$. And the respective $D^{*}$ value found using the formula (8) is equal to $5.2 \cdot 10^{-5} \mathrm{~cm}^{2} / \mathrm{s}$.

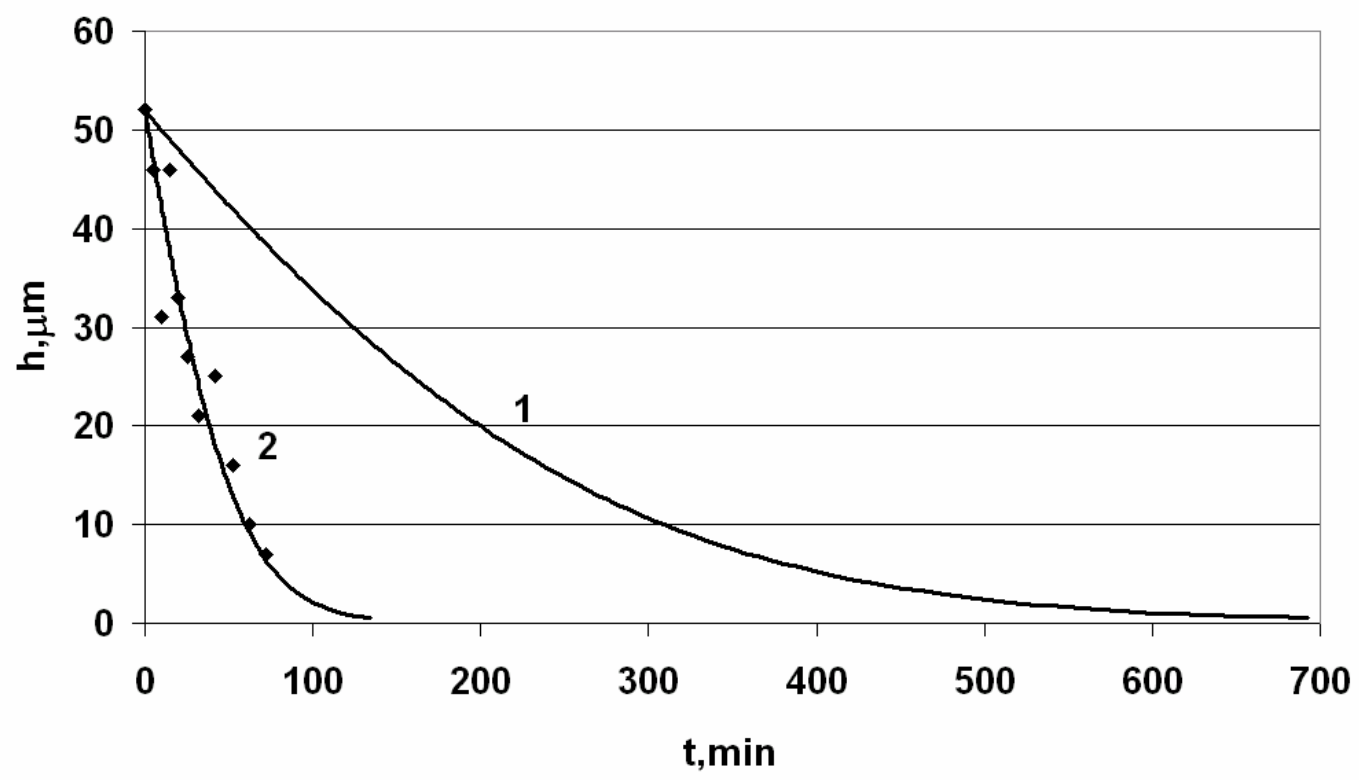

Fig. 3. Dependence of the valley depth on the time of CCMP process: 1 - calculation in accord with the formula (1); 2 - with the formula (7). 


\section{Conclusions}

1. Modelling the process that provides smoothing the irregularities on the GaAs (100) wafer surface by using the method of contactless chemical-andmechanical polishing in bromine etchant (bromine concentration equals $0.06 \mathrm{~g} / \mathrm{cm}^{3}$ ) with the rotating wafer without account of convection does not allow satisfactory description of experimental results.

2. Taking convection into account by introducing the coefficient $K$ into the formula for the "effective" diffusion coefficient $D^{*}$ has enabled to reach optimal correspondence between experimental data and calculated values (for the parameters $D^{*}=5.2 \cdot 10^{-5} \mathrm{~cm}^{2} / \mathrm{s}$ and $\left.\Delta=45 \mu \mathrm{m}\right)$.

3. For the given $\Delta$ value, total removal of the valley from the wafer surface needs etching the surface layer considerably exceeding the initial valley depth. Losses of material in the course of smoothing the surface are decreased with lowering the $\Delta$ value.
References

1. G.A. Pashchenko, M.Yu. Kravetskyi, A.V. Fomin. Modeling the process of cutting traces on semiconductor wafers under removal them by the method of chemical-mechanical non-contact polishing. Semiconductor Physics, Quantum Electronics and Optoelectronics. 2015. 18, No. 3, p. 186-189.

2. Fomin A.V., Kravetskyi M.Yu., Pashchenko G.A., Lutsyshyn I.G. Investigation of the convectivediffusion mechanism of smoothing GaAs surface irregularities by using the method of nonabrasive chemical-and-mechanical polishing. Materials of the 6-th Ukrainian sci. conf. on physics of semiconductors (September 30 - October 4, 2013). Chernivtsi: Ruta, 2013. P. 586-587 (in Ukrainian).

3. Levich V.G. Physical-and-chemical Hydrodynamics. Moscow: Fizmatgiz, 1959. 699 p. (in Russian). 